\title{
Edge2Analysis: A Novel AloT Platform for Atrial Fibrillation Recognition and Detection
}

This paper was downloaded from TechRxiv (https://www.techrxiv.org).

\section{LICENSE}

CC BY 4.0

SUBMISSION DATE / POSTED DATE

28-01-2022 / 01-02-2022

\section{CITATION}

Wu, Wanqing; Chen, Jiarong; zheng, yingfang; Liang, Yingshan; Zhan, Zehui; Jiang, Mingzhe; et al. (2022): Edge2Analysis: A Novel AloT Platform for Atrial Fibrillation Recognition and Detection. TechRxiv. Preprint. https://doi.org/10.36227/techrxiv.19084310.v1

$\mathrm{DOI}$

10.36227/techrxiv.19084310.v1 


\title{
Edge2Analysis: A Novel AloT Platform for Atrial Fibrillation Recognition and Detection
}

\author{
Jiarong Chen, Yingfang Zheng, Yingshan Liang, Zehui Zhan, Mingzhe Jiang, Xianbin Zhang, Daniel S. da \\ Silva, Wanqing Wu*, Victor Hugo C.de Albuquerque, Senior Member, IEEE
}

\begin{abstract}
Atrial fibrillation (AF) is one of the most frequently diagnosed arrhythmia in clinical practice. Recent years have witnessed an upward trend in the diagnosis of atrial fibrillation, demanding automated atrial fibrillation analysis to release the burden on health care resources. However, most automated ECG analysis tools for AF detection are implemented on large-scale equipment, which might cause problems such as high resource consumption, slow response, and privacy disclosure. Regarding the issues above, edge computing can be introduced in atrial fibrillation detection. This paper proposed an individualized atrial fibrillation detection device adopting edge computing and the neural network. First, this paper selects five features by comprehensively considering correlation, variance and computational complexity. Next, the initial neural network model is trained on non-target receptors and downloaded from the internet to the device. The finetuning training and inference process can be completed both on the device in practical application. This paper has tested the initial model to achieve an F1-score of $91.6 \%$ in CinC2017 and $93.2 \%$ in CPSC2018, and model performance could be improved after personalized modification since the edge device can retrain the model by modifying the training data set. The results show that our system has well completed AF detection and classification tasks, with the advantages of low resource consumption, fast response, and effective privacy protection. It is applicable for various healthcare environments such as military, rural areas and elderly communities.
\end{abstract}

Index Terms-Atrial Fibrillation, ECG Automated Classification, Edge Computing, Model Retraining.

\section{INTRODUCTION}

A TRIAL fibrillation, often named AF briefly, is a common arrhythmia disorder associated with high mortality and morbidity in many cardiovascular diseases. For example, about

Asterisk indicates corresponding author. Wanqing $\mathrm{Wu}$ is with the School of Biomedical Engineering, Sun Yat-sen University, Guangzhou, China. (e-mail: wuwanqing@mail.sysu.edu.cn)

This work was supported in part by the National Natural Science Foundation of China under grants (61873349); the General Logistics Department of PLA (BLB19J005); and by the Guangzhou Science and Technology Planning Project (202003000040).

Jiarong Chen, Yingfang Zheng, Yingshan Liang, Zehui Zhan, Mingzhe Jiang and Xianbin Zhang are both with the School of Biomedical Engineering, Sun Yat-sen University, Guangzhou, China. (e-mail: chenjr56@mail2.sysu.edu.cn, zhengyf57@mail2.sysu.edu.cn, liangysh28@mail2.sysu.edu.cn, zhanzh6@mail2.sysu.edu.cn, jiangmzh6@mail.sysu.edu.cn, zhangxb55@mail2.sysu.edu.cn.)

Victor Hugo C. Albuquerque and Daniel S. da Silva are both with Universidade de (e-mail: Fortaleza.victor.albuquerque@unifor.br, danielssilva@alu.ufc.br) a third of all strokes can be related to atrial fibrillation [1] [2]. According to World Health Organization, about 90 million people have atrial fibrillation worldwide, accounting for 1 to 2 percent of the world's population [3] [4]. More than 7.9 million people are suffering from atrial fibrillation in China, and the number of patients is increasing with age [5]. Atrial fibrillation can be subdivided into paroxysmal AF, persistent $\mathrm{AF}$, and permanent AF [6]. Although paroxysmal atrial fibrillation may sometimes have palpitations, chest tightness and panic symptoms, it is difficult to be perceived by patients and even doctors in most cases due to the non-specificity of the symptoms. Notably, paroxysmal atrial fibrillation may occur with nearly normal ventricular rate and have no apparent symptoms at all. Due to subjective unawareness, a large proportion of patients with atrial fibrillation do not receive timely treatment until more severe complications occur (such as atrial fibrillation with heart failure, atrial fibrillation with stroke, etc.). However, the consequence of delayed treatment is that paroxysmal AF may evolve into permanent AF. Therefore, detecting AF in the early stage plays a key role in preventing atrial fibrillation from worsening as well as subsequent complications from developing.

Electrocardiogram(ECG) plays a significant part in AF detection and diagnosis, depicting the differences between normal and atrial fibrillation signals in both atrial activity (represented by $\mathrm{P}$-wave and f-wave) and ventricular response (represented by QRS complex) [7]-[9]. AF patients mostly have ECG recordings characterized by irregular variability of $\mathrm{RR}$ interval or P-wave absence. Based on this knowledge, artificial intelligence technology has been exploited to automatically identify AF from massive ECG data to replace the tedious and time-consuming manual interpretation work. The interpretation of ECG poses a great challenge to the medical practice, with about 3 million electrocardiograms being recorded each year worldwide. Therefore, automated ECG interpretation must be studied. Machine learning, one of the outstanding approaches, is carried out and proved the model can perform as well or even better than cardiologists [10]. Recent studies focusing on machine learning have yielded some encouraging results. Deep Convolutional Neural Networks (CNN) with multi-layers or densely connected structures are used by many researchers [1], [11][13]. Furthermore, merged with Recurrent Neural Networks (RNN), Limam et al. [14]based their classification works on a convolutional recurrent neural network (CRNN). Other hybrid 
neural network architectures (such as CNN-LSTM) were also designed by Andersen R S et al. [15] and Petmezas $\mathrm{G}$ et al. [16] to integrate the process of feature-extracting and modelinferencing. In addition, Dual-channel Neural network is also proposed in [17] for AF detection, which uses time-frequency spectrum and Poincare plot of the denoised ECG signal.

The disadvantages of the above methods are obvious. It is known that these researches have a great achievement on the ECG classification task at the tremendous cost of data transfer, computational complexity and response delay. These problems are especially serious in underdeveloped countries, remote rural areas, or special areas, such as elderly communities and military commands, that either cannot support adequate online resources or require strict secrecy. The disadvantages of the above methods are obvious. Taking rural areas as an example, the number of heart attacks and deaths is more considerable than urban areas on account of poverty, harsh living conditions and scarce healthcare resources, appealing more cost-effective and practicable methods of AF detection. Some researchers have floated the idea of combining atrial fibrillation detection tasks with wearables [18] or smartphones [19] to give users real-time health information. Moreover, a research [20] suggests a platform where doctors can monitor and monitor patients' health remotely and users can monitor their own care using personal devices, which may be the dominant approach for chronic disease care in the future. As a key answer to that question, edge computing should be introduced. By processing data locally, we can only use a few resources to implement primary screening for atrial fibrillation, and it can be supposed that cost reduction associated with screening for AF will facilitate the government to make healthcare decisions. Even only in terms of property, it is necessary to take time to study edge computing.

The definition of edge computation is pushing traditional applications closed to where the data is generated [21], which calls for local processing of the data from users [22]. In addition, it has the potential to complement the shortcomings of the cloud computing model. For one, edge computing is always related to high response speed, low cost and data security [23]: personal data just need to be stored in the edge devices instead of uploading to the cloud, eliminating the problem of data leakage fundamentally. For another, the edge computing algorithm requires a large number of computing resources [24], which is a very difficult problem for resourceconstraint [25] embedded devices to implement. Many scholars have studied the implementation of edge computing, the work [26] realizes a tiny neural network for environmental predictions, but the memory consumption is acceptable, and model prediction is more accurate than traditional methods. Some researchers [27] are applying it to healthcare, where devices can better serve patients by directly detecting their voice to determine their health degree. Another research [28] that focuses on real-time is the Human Detection task, proposing a lightweight $\mathrm{CNN}$ deployed on the edge node and yielding satisfactory results. The remarkable literature [25] adopts a new framework-ML-MCU, which enables edge devices to train binary and multi-classification models and solves the resource limitation problem. It is also common for edge computing to be applied to the processing of physiological signals. For example, researchers [29] designed a CNN chip to complete the emotion recognition task at the edge.

Concluding from these works, there are two ways to implement complex computing tasks on edge devices:(1) Use specific chips, like AI chips[26], which are designed for edge computing. (2) Simplify the task by reducing the calculated amount. In the process of ECG processing, Feature Engineering (FE)-based input is obviously more suitable than the original signal for edge calculation, so one device is used to complete feature engineering, and another device would run the neural network, the equipment can perform less calculation in this way. In this work, we have designed a neural network that can be used for ECG classification tasks and then implemented it on edge devices. Unlike other works, we make it possible to directly train the new model at the edge. In other words, to meet the specific physiological signals, the model can be individual. Although the initial model is the same, the acquisition of a personalized specific model can be made with the assistance of an expert. The main contributions of this paper are as follows:

- We selected suitable features according to the characteristics of edge devices so as to complete the AF classification task, and the $F_{1}$ score of this model on the test-set was about $90 \%$.

- Our model can be implemented on embedded devices, without any participation of the cloud, edge devices is enough to finish signal collection, processing and result analysis.

- The model proposed in this paper can be personalized based on their own ECG signals and corresponding labels, and this paper experimentally investigate the importance of initial models and initial data sets.

The rest of this paper is organized as follows. Section II will introduce the main dataset used in training and testing work. On this basis, the definition and calculation of five features extracted from ECG signals are also presented in this part. Through the perspective of the algorithms, Section III depicts the signal flow and transformation, neural network's function method and the operational modes switch between inferencing and retraining. Next, a series of progressive experiments and results discussions are presented in Section IV with the evaluation indicators and retraining strategies proposed too. The superior performance in both model train simulation and realtime inference can validate our method and design's feasibility. Finally, Section V will briefly summarize our work and give a vision for the future.

\section{DATA PrEparation}

The dataset we used in the progress of network training and testing is obtained from the Computing in Cardiology Challenge 2017 [30], whose openly available training set contains 8,528 single-lead ECG recordings lasting from 9s to 60s with a sampling rate of $300 \mathrm{~Hz}$. The ECG signals whose recording length is $30 \mathrm{~s}$ are chosen and the relevant information is shown in Table I, $40 \%$ for training, $40 \%$ for testing and $20 \%$ for real-time simulation. Since our project focuses on the realtime detection of AF from normal ECG signals, we utilize the 
Normal and AF ECG recordings in our study. Some of the CinC2017 are chosen as the training dataset and another part as the test dataset. This network could be tested on the dataset of the China Physiological Signal Challenge 2018(CPSC 2018) [31]. Sampled at $500 \mathrm{~Hz}$, CPSC 2018's public training data involve 6,877, 12-lead ECG recordings lasting from 6s to 60s. In our work, 447 AF-type ECG recordings and 454 normaltype ECG recordings derived from the second lead are selected to compose a heterogeneous dataset, whose signal length is $15 \mathrm{~s}$.

TABLE I

DATA PROFILE FOR TRAINING AND TESTING SET

\begin{tabular}{ccccc}
\hline Dataset & Sample Frequency(Hz) & Normal & AF & Total \\
\hline CinC 2017 & $300 \mathrm{~Hz}$ & 500 & 500 & 1000 \\
CPSC 2018 & $500 \mathrm{~Hz}$ & 447 & 454 & 901 \\
\hline
\end{tabular}

A prominent characteristic of AF is the irregularity of RR interval (RRI), and due to R-wave's large amplitude and drastic changes in QRS complex slopes, AF detection based on $\mathrm{R}$ waves and RR interval is more robust compared with other segments.
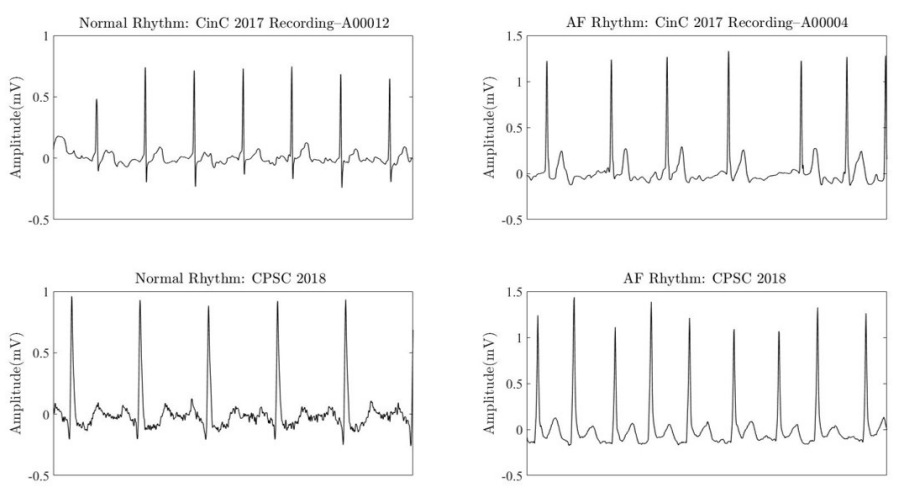

Fig. 1. Atrial Fibrillation Recording and Normal ECG Recording in two dataset.

Hence, the main steps include detecting R peaks' positions, obtaining the RR interval between two beats, and calculating effective features as the inputs for training and inference. In $\mathrm{R}$ peaks detection, we refer to the Pan-Tompkins [32] algorithm and Arduino Heart Rate Analysis toolkit [33]. Derived from the information of RR interval, it is possible to calculate statistical features that can discriminate AF and Normal categories. Based on RRI's irregularity theory and capabilities of embedded devices, we selected some HRV-based features [8] [9] [34] [35].

\section{Application Scenarios}

The most important part this paper proposed is how to design the Atrial Fibrillation System. The realization of neural network has been very mature. The multilayer perceptron is implemented in embedded devices, it is known that the size of hidden neurons had better be set between that of the input and the output neurons [36]. In addition, the method of training model is gradient descent with momentum [37]. Getting the public data set online for free and then the initial model is obtained and would like to be transplanted in the embedded device. It is worth noting that this part of the work can be done on the toolbox of MATLAB, and we will prove this experimental idea. Moreover, our research focuses on the evolution of the model to meet the personalized demands, which is done on the embedded device side. In this part, we mainly illustrate the realization process of the system from the perspective of the algorithm. The main function is shown in Algorithm 1. Determined by its control signal, it can complete model inference or model retraining.

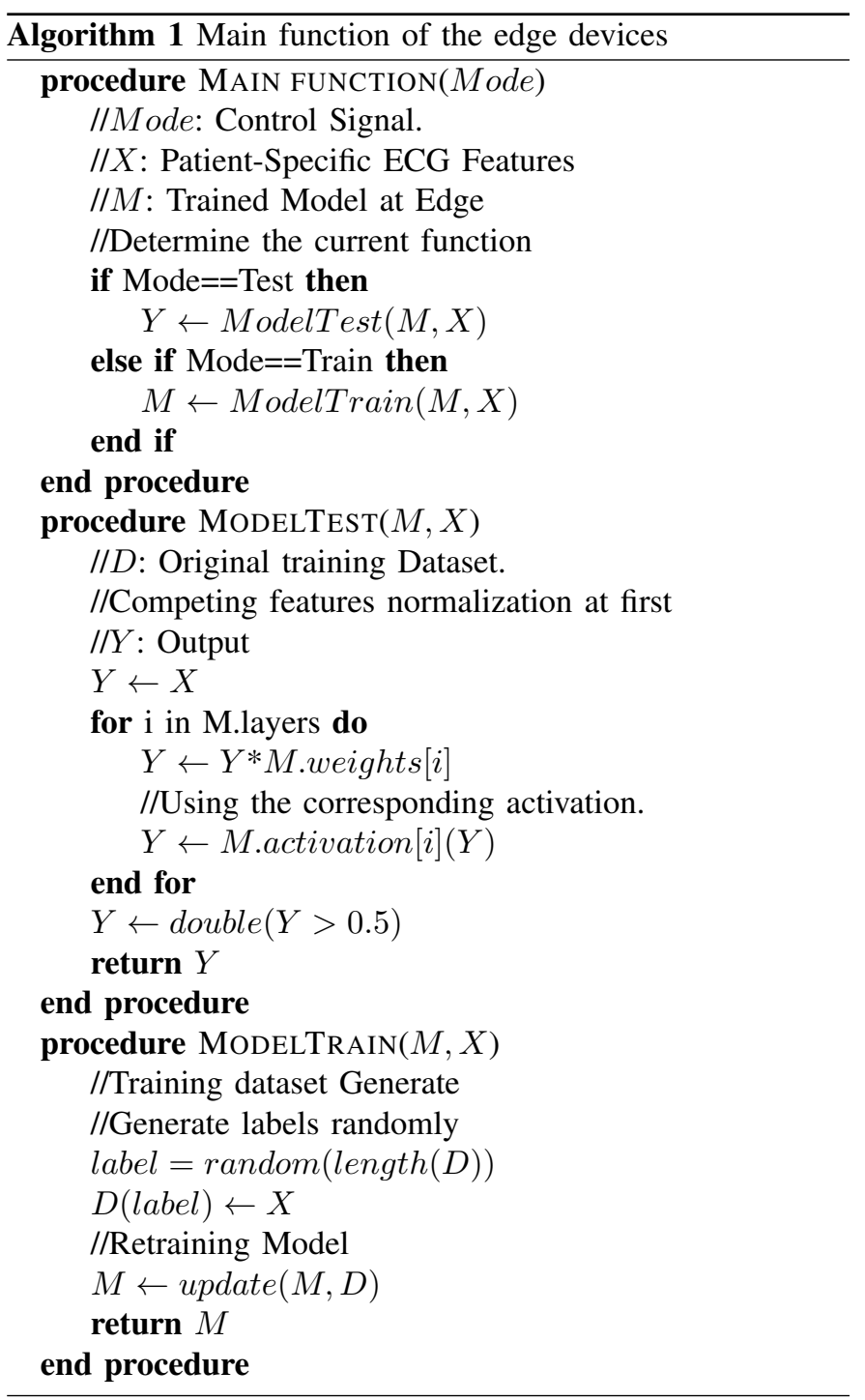

Considering that the data are divided into two categories, it is possible for the data to be extremely unbalanced and the model performance to be extremely degraded. Therefore, we divided the reference data into two parts according to categories, and the new data will replace the data in the same category with it. In addition, feature extraction also ought to be introduced.

Figure 2 greatly indicates the functions of the system designed in this paper, which can collect the ECG signals from users through three electrodes, then the raw data should 


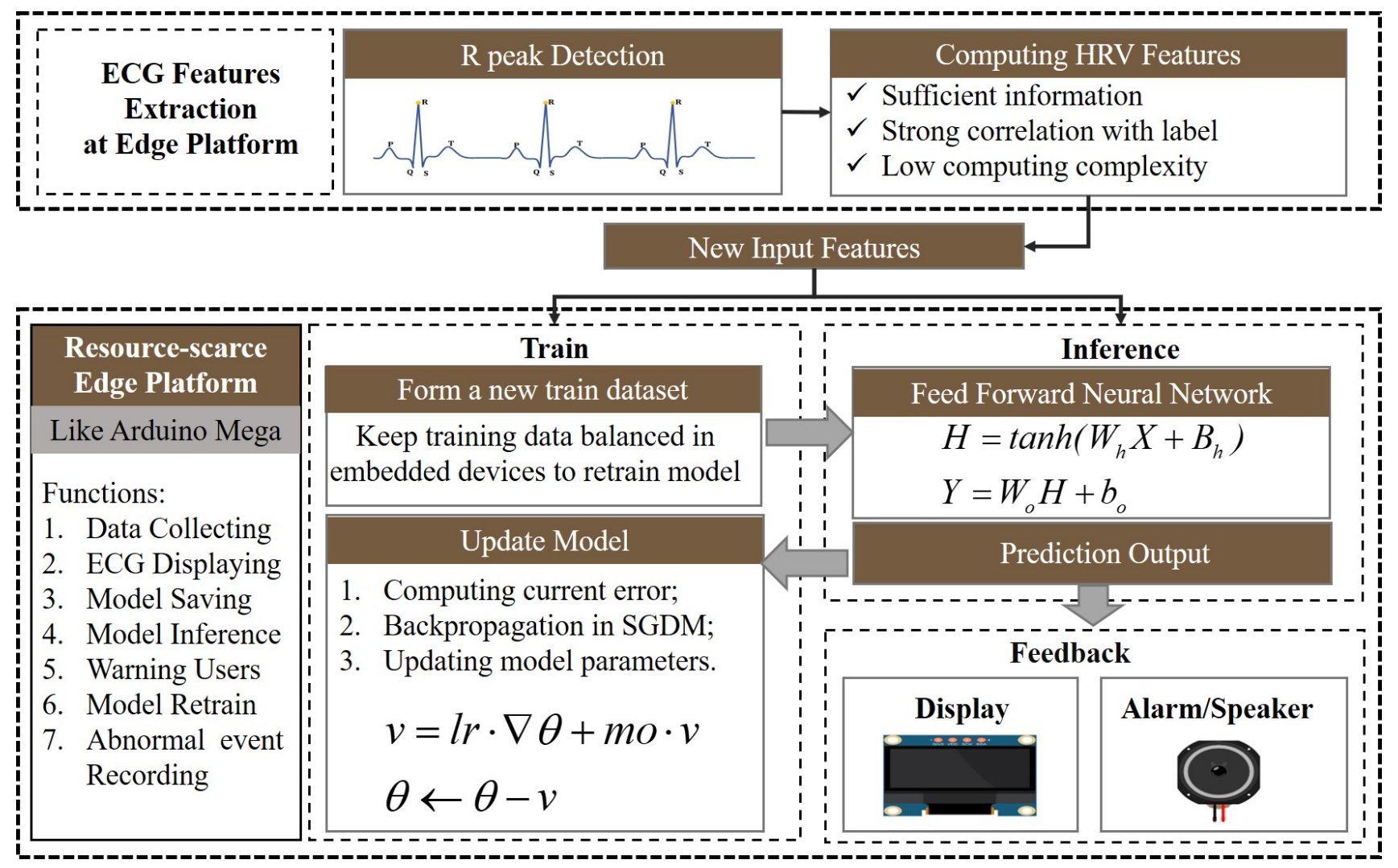

Fig. 2. ECG-based classification system schematic diagram. The original ECG signal is obtained through the sensor, and then the feature is extracted. The system can implement training or inference according to the actual situation

be filtered to offset the noise effect. Therefore, the processed signal is more suitable than the original signal to perform Rwave recognition and feature extraction. Then we will feed the features into the BP neural network for classification. If the data is labeled, we can also conduct training, which will be described in detail in the next section. The output can also be directly displayed, which helps patients understand their physiological state. A brief demonstration is shown in Figure 2, the mathematical principles, including the calculation of the features, the design of the neural network model, and how the edges update the model. The hardware we utilize in our work includes Arduino Mega, Arduino Nano, OLED display, speaker, AD8232, ECG signal generator SKX2000sup+, button and power supply. The designed system adopts accessories including Arduino Mega, Arduino Nano, OLED display screen, Speaker, AD8232, integrated triple lead wire, ECG electrode patch, button and power supply. In this system, we have two operational modes. One is the AF detecting mode based on the trained neural network, and the other is the retraining mode to update the model based on the specific user's signals. The ECG signal is transmitted to AD8232 through the lead wire as the front-end processing. Then the feature-extracting and neural network inference are carried out inside Arduino Mega. The judge result (atrial fibrillation or normal, represented as high or low level) is transmitted to Arduino Nano, to control what the OLED display and whether speakers warn.

On top of that, it is easy to switch to the retraining mode through the button to optimize the neural network model to a specific individual. After measuring a piece of individualized ECG signal and extracting sufficient features for network judgment, it needs the correct label at the moment (normal or atrial fibrillation) to be entered manually, which can be available with the help of medical practitioners. We will use the ECG signal generator SKX-2000sup+ to simulate atrial fibrillation and a normal ECG signal to optimize the network model. In addition to the above equipment, the rest of the kit includes a speaker, power supply, buttons that switch operational modes of the system. It is worth mentioning that due to the relatively low hardware cost, it can also serve the vast underdeveloped areas.

\section{Results ANd Discussions}

\section{A. Experimental Environment}

The classification model runs on MATLAB and Arduino respectively. MATLAB is deployed on a personal computer, which is equipped with an Intel (R) Core (TM) I5-9400, with RAM up to $8.00 \mathrm{~GB}$. The edge devices we use are Arduino Uno and Arduino Mega, with ATmega328 and ATmega2560 processors, and their storage space and computing capacity are inferior to that of computers. In the following experiments, we will complete the selection and training of the initial model on MATLAB and deploy it on the edge device. 


\section{B. Classification Performance}

To evaluate the experimental results reasonably and objectively, the corresponding evaluation system must be built. Generally speaking, for a binary classification task, there are many evaluation indexes that can be used, including CrossEntropy (CE) [38], Mean Square Error (MSE), accuracy [39] and other indexes. Accuracy can tell what percentage of all samples are correctly distinguished, which is shown in the following formula.

$$
a=\frac{T P+T N}{T P+F P+F P+F N}
$$

In this work, we mainly chose the $F_{1}$ score, which is derived from the positive precision and recall. The common concepts should be well known. For example, it is normal to regard fewer categories as positive categories in classification problems and name more categories as negative ones. Therefore, in this study, Atrial Fibrillation is the positive category while normal ECG is the negative category. For a special classifier, the prediction can not always be equal to the actual situation. When the prediction is positive, and the actual result is also positive, this situation will be called TP. The prediction accuracy of the positive category is expressed by $p$ and is shown in the following formula.

$$
p=\frac{T P}{T P+F P}
$$

The variable $p$ mentioned in the above equation is mainly used to evaluate the correct rate of the classifier to the positive category, that is to say, how many samples out of all the samples predicted by the classifier to be the positive category belong to the real positive category? Another indicator that needs to be introduced is recalled rate $r$, which is defined as follows:

$$
r=\frac{T P}{T P+F N}
$$

Different from $p, r$ is more concerned about whether the classifier designed by us can detect all positive signals. For comprehensive consideration, we use $F_{\beta}$ score, which is defined as shown in the figure below:

$$
F_{\beta}=\frac{\left(1+\beta^{2}\right) p r}{\beta^{2} p+r}
$$

To evaluate the performance of the classifier more objectively and accurately, the $F_{\beta}$ is usually used in the classification task. As a regulating item, $\beta$ can be used to distinguish the importance of $p$ and $r$. In general, we consider these two indicators equally important, so $\beta$ equals one. At the same time, we can call $F_{\beta}$ the $F_{1}$ score.

\section{System Implementing}

In this section, the experimental setup needs to be introduced. First, we need to do a preliminary experiment, which is used to select features. The following experiments involve exploring the appropriate initial model, real-time edge inference and the model retraining simulation. In the experiment process, the number of normal and AF signals were equal, which effectively ruled out the effects of data imbalance. In addition, repetitive experiments are done to obtain general results. Real-time edge inference experiments can verify the model performance in real scenarios and lay a foundation for further design in the model update. In this experiment, we utilize the ECG signal generator to get real-time raw data to supplement the signals obtained from the non-AF subjects. Inference on edge devices is a necessary condition for realtime retraining. Retraining simulating the actual situation of this system is a must.

1) Feature Selecting: In the field of machine learning, feature selection [1] is of great importance, but in this paper, we must consider the factors which are brought by embedded devices. In the process of feature selection, three factors are chosen: large information, strong correlation with labels (and weak correlation in features) and low computational complexity. The front factors can be easily found in relevant research, but the low computational complexity is rarely mentioned for abundant computing resources in computers. However, edge devices usually have insufficient resources. In the calculation of correlation features, there are three main processes: multiplication (division), addition (subtraction) and numerical judgment. Our estimation results of the computational complexity of related features are shown in Table II.

\begin{tabular}{|c|c|c|c|c|}
\hline Domain & Feature & Complexity $^{1}$ & Feature & Complexity \\
\hline \multirow{6}{*}{ Time } & MRR & $\mathrm{O}(1)^{2}$ & MeanHR & $\mathrm{O}(1)$ \\
\hline & NN20 & $\mathrm{O}(1)$ & PNN20 & $\mathrm{O}(1)$ \\
\hline & NN50 & $\mathrm{O}(1)$ & PNN50 & $\mathrm{O}(1)$ \\
\hline & SDNN & $\mathrm{O}(\mathrm{N})$ & SKEW & $\mathrm{O}(\mathrm{N})$ \\
\hline & SDSD & $\mathrm{O}(\mathrm{N})$ & RMSSD & $\mathrm{O}(\mathrm{N})$ \\
\hline & CVRR & $\mathrm{O}(\mathrm{N})$ & CVdRR & $\mathrm{O}(\mathrm{N})$ \\
\hline \multirow{3}{*}{ Frequency $^{3}$} & ULF & $\mathrm{O}\left(N_{1} \log \left(N_{1}\right)\right)$ & VLF & $\mathrm{O}\left(N_{1} \log \left(N_{1}\right)\right)$ \\
\hline & LF & $\mathrm{O}\left(N_{1} \log \left(N_{1}\right)\right)$ & $\mathrm{HF}$ & $\mathrm{O}\left(N_{1} \log \left(N_{1}\right)\right)$ \\
\hline & $\mathrm{LF} / \mathrm{HF}$ & $\mathrm{O}\left(N_{1} \log \left(N_{1}\right)\right)$ & $\mathrm{TP}$ & $\mathrm{O}\left(N_{1} \log \left(N_{1}\right)\right)$ \\
\hline \multirow[t]{2}{*}{ Nonlinear } & SD1 & $\mathrm{O}(\mathrm{N})$ & SD2 & $\mathrm{O}(\mathrm{N})$ \\
\hline & SD1/SD2 & $\mathrm{O}(\mathrm{N})$ & CovRR & $\mathrm{O}(\mathrm{N})$ \\
\hline
\end{tabular}

TABLE ॥

ESTIMATION OF COMPUTATIONAL COMPLEXITY FOR HEART RATE VARIABILITY CHARACTERISTICS

${ }^{1}$ Since the running time of multiplication (division) is longer than that of addition (subtraction) and judgment. For a fixed RR sequence, we mainly consider the operations time of multiplication and division in feature calculation.

${ }^{2} \mathrm{O}(1)$ represents a finite number of multiplications during feature calculation and is independent of sequence length, usually less than $\mathrm{O}(\mathrm{N})$.

${ }^{3}$ Interpolation of RR interval is a routine process in the process of calculating frequency-domain feature and $N$ in actual estimation should be the interpolated sequence length $N_{1}$, usually equals $f s \times N$.

Therefore, it can be known that the computational complexity of frequency-domain features of heart rate variability is much higher than that of time-domain features and a number of nonlinear features for the estimation time in the feature calculation process. In addition, frequency domain features also require more spatial memory due to the interpolation of RR intervals. In the next, calculate the variance and label correlation of the features respectively. The results are shown in Fig 3.

Under the constraint of computational complexity, we hope the features can have enormous information and strong correlation with the label. In addition, among the features with strong correlation, we deleted the features with less information. 

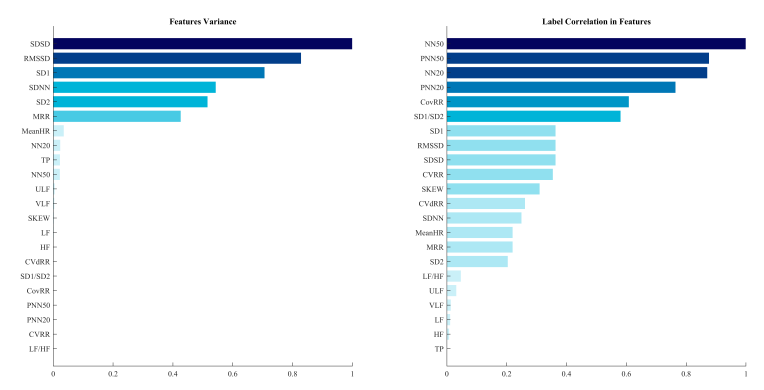

Fig. 3. The variance and label correlation of the features, and the left represents the variance of the features of the training data set, while the right shows the correlation between features and labels.

However, due to the strong linear correlation of some features, we conducted linear correlation analysis for all features in Fig 4. Among features whose correlation was greater than $70 \%$, only one feature was saved.

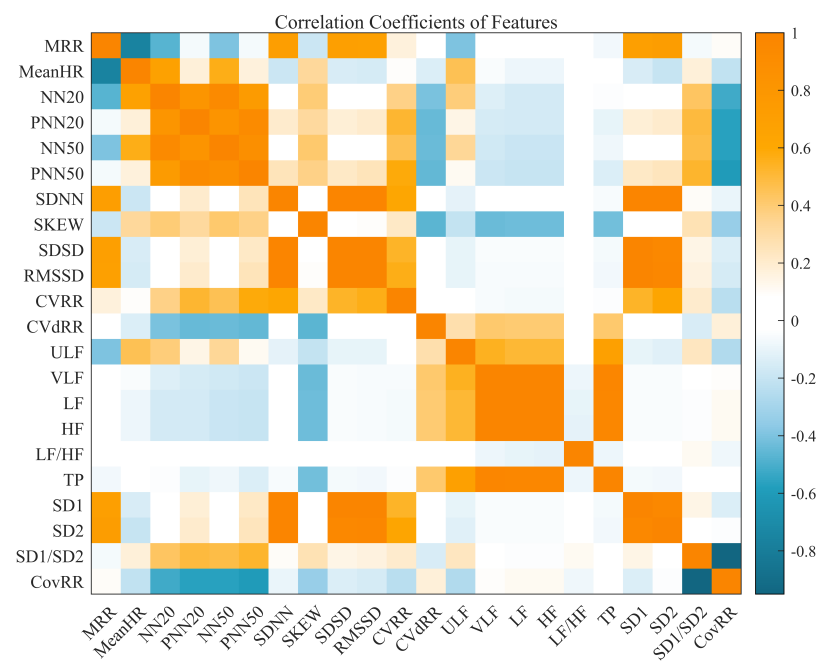

Fig. 4. Correlations between heart rate variability features. The higher the value, the higher the correlation between features, and highly relevant features can be purposefully removed.

Given these factors, the features we choose are MRR, PNN50, SDSD, Skew and covRR. The following work is also focused on using these features. As far as we know, we are the first in the field of edge computing to propose feature selection for the attributes of embedded devices.

2) Initial Model Training: Previously, we have selected the required features through some criteria. Here, The training of the initial model was mainly completed on MATLAB, and the termination condition was that the training times reached 5000 times, using 5-3-1 three-layer neural network model structure. The test results are shown in Fig 5.

Experimental results in Fig 5 show that the initial model based on feature engineering training has excellent performance, and its prediction accuracy exceeds 90\%. The error bar was made in Fig 5, which shows that the training process of this model has strong repeatability and proves the feasibility of feature engineering. The relationship between the amount of data and model performance is also a factor to be considered in

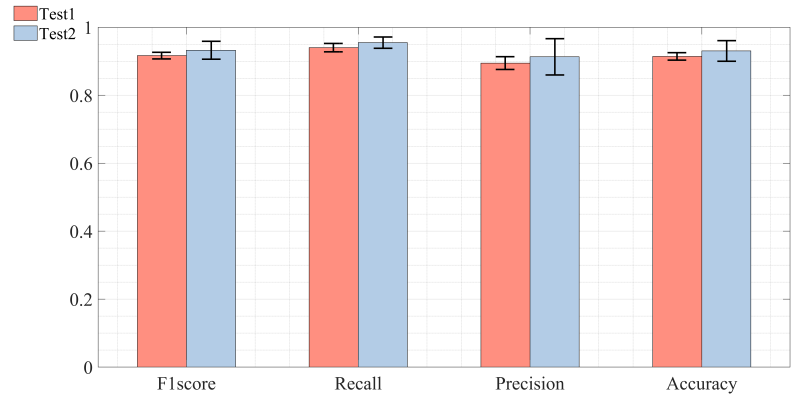

Fig. 5. Initial model tests performance. The test sets are from CinC2017 and CPSC2018 respectively

this study. In order to obtain better performance, it is necessary to have a complex model and a large amount of data. If the relationship between the two can not be adjusted reasonably, it may lead to under-fitting or over-fitting. In this study, we must consider the limited resources of embedded devices, so the initial dataset in embedded devices should not be too large. In our previous work, we found that the amount of data that embedded devices can train is limited, generally less than 100 . Since this paper expects that the edge devices can continuously train the model, and the model can learn both public and private patterns, it is recommended to select approximate data with sufficient information to achieve stable and outstanding model performance.

3) Real-time Inference Simulation: Due to the lack of clinical ECG signals actually collected, we constructed a simulation dataset in this paper. In this section we will use this data set for model testing, and in the next section we will also use this dataset for model retraining. The test results of 200 samples are shown in the table below.

TABLE III

Model Performance Simulation of Real-time Testing

\begin{tabular}{ccccc}
\hline Attribute & $F_{1}$ & $p$ & $r$ & $a$ \\
\hline Value & 0.88 & 0.83 & 0.94 & 0.87 \\
\hline
\end{tabular}

Through three test sets, it can be found that the model has good generalization ability. However, the amount of data used in this experiment is not real-time. We should try to obtain data from clinical sources in the future. In addition, we designed an experiment to prove the importance and the advantage of edge computing, such as low response time and low power consumption. In this experiment, edge devices are just used to collect ECG signal and send the original signal to the computer for processing through a serial port (or other communication approaches). The experiment was conducted with 150 samples at a time, and the response time is computed, which does not include the time of feature extraction, and the experimental result is shown in Table IV.

In Mode 1, data is stored on the server and transmission is not required. However, it is difficult to implement it in wearable form because it requires direct connection to the server. Mode 2 is that the index data is collected by the embedded devices and then sent to the server for processing, so its response time is the calculation time and transmission time. 
TABLE IV

COMPARISON OF THREE DATA PROCESSING MODES.

\begin{tabular}{ccccc}
\hline Mode & Data Source & Data Processing & Wearable & Response time \\
\hline Mode 1 & Server $^{1}$ & Server & $\mathrm{N}$ & $0.11 \mathrm{~ms}$ \\
Mode 2 & Edge & Server & $\mathrm{Y}$ & $6.13 \mathrm{~ms}$ \\
Mode 3 & Edge & Edge & Y & $2.12 \mathrm{~ms}$ \\
\hline
\end{tabular}

${ }^{1}$ In this study, a personal computer is used as the server, which has much more computing power than edge devices. Data belonging to this table may change on other server devices. Since the server can compute in parallel, the sample size has little effect on the response time in Mode 1.

Mode 3 is the edge computing mode which is studied in this paper, it is proposed for the first two modes require the participation of the server, the cost is high and easy to be limited by network conditions. Through the comparison of three data processing modes, some conclusions can be obtained: from the perspective of computing, the server processing speed is faster than the edge devices, and the server is capable of parallel computation. When the transmission process is considered, then the direct processing at the edge devices can obtain lower latency and lower power consumption, and more importantly, can effectively eliminate the risk of privacy leakage. The results of Table IV indicate the feasibility and relatively high accuracy of edge inference, which is the basis for further design of individualized lightweight neural networks at the edge device.

4) Model Retraining: If the device fails to judge the target data from a specific user, the cost is high, so we develop the strategy of retraining and updating the model. The model performs poorly on some patterns that it has not observed before. Experimental results show a pattern: in parallel experiments, the samples that fail to recognize each time are highly overlapped. The initial training dataset was mentioned earlier because the training dataset can be updated in real-time. The reason this article uses a combination of old and new data rather than just new data is used to train model because we want the model to see more patterns. In order to study the real-time training model of embedded devices, we specially designed this experiment. The experiment requires that the new input data can replace a sample in the training dataset, which is used in this paper is category replacing method (CR). The $F_{1}$ score is chosen as the evaluation metrics. In the first experiment, we studied the significance of the model retraining process. The size of the original dataset was 100 , and the size of the new dataset was 20 . The number of normal and abnormal signals in both data sets is equal. We conducted three groups of experiments, namely group $\mathrm{N}$ (original model), A (Without initial dataset and without trained model), B(without initial dataset but with trained model), and $\mathrm{C}$ (with initial dataset and trained model), the relevant experimental results is shown in $\mathrm{V}$.

Table V only discusses a simple case, So there's not much difference between groups, but it is known that models based on trained model generally improved training speed. Which we also need to study the impact of the total number and proportion of new data on the model performance. Considering clinical practice scenarios, the category of ECG belonging to a user is not necessarily homogeneous, and the number of new
TABLE V

NEW MOdEL TRAINING PERFORMANCE WITH DIFFERENT EXPERIMENTAL GROUP

\begin{tabular}{ccccc}
\hline Group & $F_{1}$ score & $a$ & $p$ & $r$ \\
\hline N & $0.9153 \pm 0.0085$ & $0.9118 \pm 0.0099$ & $0.8820 \pm 0.0197$ & $0.9517 \pm 0.0120$ \\
A & $0.9083 \pm 0.0058$ & $0.9045 \pm 0.0065$ & $0.8736 \pm 0.0098$ & $0.9460 \pm 0.0054$ \\
B & $0.9226 \pm 0.0069$ & $0.9211 \pm 0.0074$ & $0.9061 \pm 0.0126$ & $0.9399 \pm 0.0088$ \\
C & $0.9184 \pm 0.0098$ & $0.9161 \pm 0.0107$ & $0.8945 \pm 0.0194$ & $0.9441 \pm 0.0146$ \\
\hline
\end{tabular}

data should not have a limitation. In order to control variables and contrast with Table $\mathrm{V}$, normal signals and AF are evenly distributed when the total number changes. In the case of varying proportions, we can show the experimental results of a new dataset of 20 samples. Considering the imbalanced data, F1 score is selected to present the results in this experiment, and note that this is the average of 100 replicates.

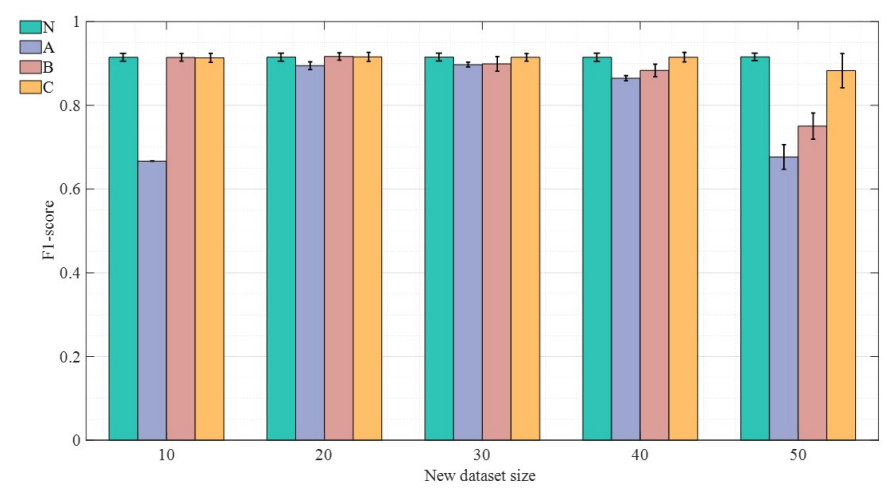

Fig. 6. Model Performance Comparison with new dataset in Three Groups

It can be found that in the case of new data balancing, the pre-trained model can play a very important role, in which group $\mathrm{N}$ is the initial model for comparison. The results were even more pronounced when the sample size was 10, although only new data were used, the performance of group B and group c surpassed greatly than group A in the same iterations due to the pre-training model.

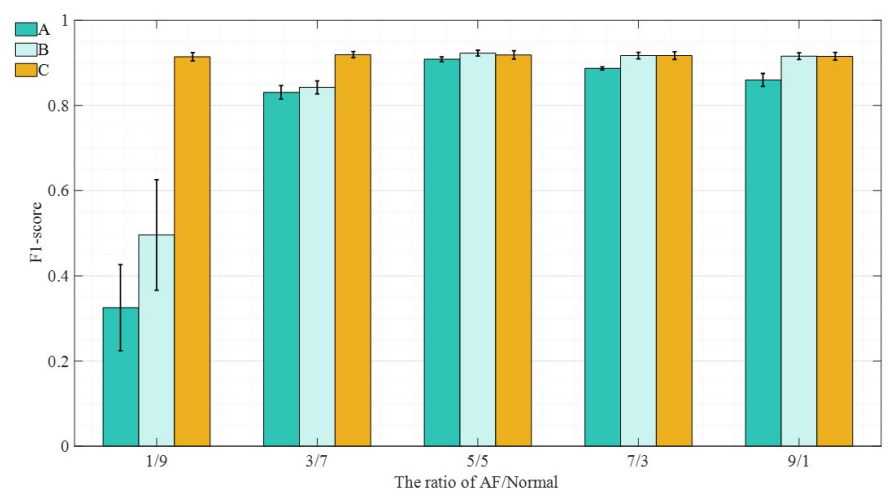

Fig. 7. Model Performance Comparison with different ratio in Three Groups

At this point, the ratio of atrial fibrillation to normal signal in the new dataset was $1 / 9$, which showed the greatest 
difference. Although group B had the original model, it still performed poorly, which proved the significance of keeping the original dataset for model retraining, even it happens with space needed in embedded devices.

\section{SUMMARY}

This paper designs an edge AF detection system based on the embedded platform and mainly uses feature engineering. The embedded system has the edge computing ability, involving collecting ECG signals in real-time and AF detecting. Moreover, our system can train a specific model based on the ECG signal of a specific user. In addition, we also studied the updating method of the training dataset. In the case of extremely unbalanced data distribution, the category replacing method (CR) is recommended to use based on the initial dataset and trained model, which is beneficial to the new training process.

Therefore, the significance of this system is outstanding. To our best knowledge, it is important to research the possibility of model evolution right on the edge platform, which has a relatively broad application prospect. This study lacks clinical data verification, cooperating with hospitals and trying multiclassification tasks can be done in the future. It can also be implemented in areas short of healthcare resources, such as rural areas, allowing more patients with atrial fibrillation to be detected and sent into the hospital for timely diagnosis and immediate treatment.

\section{REFERENCES}

[1] A Wc, B Yc, B Jg, B Bh, B Ys, J. B. Lei, C Jw, E Gzd, and A Jl. Accurate detection of atrial fibrillation from 12-lead ecg using deep neural network. Computers in Biology and Medicine, 116:103378-, 2020.

[2] L. Friberg, Mrten Rosenqvist, A. Lindgren, A Terént, and K. Asplund. High prevalence of atrial fibrillation among patients with ischemic stroke. European Journal of Neurology, 22(9):64-e5, 2014.

[3] Monique and Young. Atrial fibrillation. Critical care nursing clinics of North America, 2019.

[4] C. Yao, W. Xiao, J. Yonghan, A. Vida, Z. Ramin, B. Marvi, and A. Mohammad. Classification of short single lead electrocardiograms (ecgs) for atrial fibrillation detection using piecewise linear spline and xgboost. Physiological Measurement, 39, 2018.

[5] Xin Du, Lizhu Guo, Shijun Xia, Jing Du, and Changsheng Ma. Atrial fibrillation prevalence, awareness and management in a nationwide survey of adults in china. Heart (British Cardiac Society), 107(7):heartjnl2020-317915, 2021.

[6] F. Witassek, A. Springer, L. Adam, S. Aeschbacher, and M. Schwenkglenks. Health-related quality of life in patients with atrial fibrillation: The role of symptoms, comorbidities, and the type of atrial fibrillation. PloS one, 14(12):e0226730.

[7] S. Hong, Y. Zhou, J. Shang, C. Xiao, and J. Sun. Opportunities and challenges of deep learning methods for electrocardiogram data: A systematic review. Computers in Biology and Medicine, 122(5):103801, 2020.

[8] H. Yuki, F. Hamido, O. S. Lih, T. J. Hong, R. S. Tan, E. J. Ciaccio, and A. U. Rajendra. Computer-aided diagnosis of atrial fibrillation based on ecg signals: A review. Information ences, 467:99-114, 2018.

[9] A. Rizwan, A. Zoha, I. B. Mabrouk, H. M. Sabbour, and Q. H. Abbasi. A review on the state of the art in atrial fibrillation detection enabled by machine learning. IEEE Transactions on Biomedical Engineering, PP(99), 2019.

[10] H. Holst, M. Ohlsson, C. Peterson, and L. Edenbrandt. A confident decision support system for interpreting electrocardiograms. Clinical Physiology, 19(5):410-418, 2010.

[11] S. Kiranyaz, T. Ince, and M. Gabbouj. Real-time patient-specific ecg classification by 1 -d convolutional neural networks. IEEE Transactions on Biomedical Engineering, 63(3):664-675, 2016.
[12] Z. Xiong, M. K. Stiles, and J. Zhao. Robust ecg signal classification for detection of atrial fibrillation using a novel neural network. In 2017 Computing in Cardiology (CinC), 2018.

[13] J. Rubin, S. Parvaneh, A. Rahman, B. Conroy, and S. Babaeizadeh. Densely connected convolutional networks and signal quality analysis to detect atrial fibrillation using short single-lead ecg recordings. 2017.

[14] M. Limam and F. Precioso. Af detection and ecg classificationbased on convolutional recurrent neural network. In 2017 Computing in Cardiology Conference, 2017.

[15] R. S. Andersen, A. Peimankar, and S. Puthusserypady. A deep learning approach for real-time detection of atrial fibrillation. Expert Systems with Application, 115(JAN.):465-473, 2019.

[16] G. Petmezas, K. Haris, L. Stefanopoulos, V. Kilintzis, and N. Maglaveras. Automated atrial fibrillation detection using a hybrid cnn-lstm network on imbalanced ecg datasets. Biomedical Signal Processing and Control, 63:102194, 2021.

[17] Bo Fang, Junxin Chen, Yu Liu, Wei Wang, Ke Wang, Amit Kumar Singh, and Zhihan Lv. Dual-channel neural network for atrial fibrillation detection from a single lead ecg wave. IEEE Journal of Biomedical and Health Informatics, pages 1-1, 2021.

[18] Li Zhu, Viswam Nathan, Jilong Kuang, Jacob Kim, Robert Avram, Jeffrey E Olgin, and Jun Gao. Atrial fibrillation detection and atrial fibrillation burden estimation via wearables. IEEE Journal of Biomedical and Health Informatics, pages 1-1, 2021.

[19] O. Lahdenoja, T. Hurnanen, Z. Iftikhar, S. Nieminen, T. Knuutila, A. Saraste, T. Kiviniemi, T. Vasankari, J. Airaksinen, and M. Pankaala. Atrial fibrillation detection via accelerometer and gyroscope of a smartphone. IEEE Journal of Biomedical Health Informatics, pages 1-1, 2017.

[20] C. A. Gomez-Garcia, M. A. Askar-Rodriguez, and J. Velasco-Medina. Platform for healthcare promotion and cardiovascular disease prevention. IEEE Journal of Biomedical and Health Informatics, PP(99):1-1, 2021.

[21] M. Caprolu, R. D. Pietro, F. Lombardi, and S. Raponi. Edge computing perspectives: Architectures, technologies, and open security issues. In 2019 IEEE International Conference on Edge Computing (EDGE), 2019.

[22] W. Shi, C. Jie, Z. Quan, Y. Li, and L. Xu. Edge computing: Vision and challenges. Internet of Things Journal, IEEE, 3(5):637-646, 2016.

[23] M. Alrowaily and L. Zhuo. Secure edge computing in iot systems: Review and case studies. In 2018 IEEE/ACM Symposium on Edge Computing (SEC), 2018.

[24] Jianbiao Xiao, Qingsong Liu, Huanqi Yang, Jiahao Liu, and Jun Zhou. Ulecgnet: An ultra-lightweight end-to-end ecg classification neural network. IEEE Journal of Biomedical and Health Informatics, PP(99):1-1, 2021.

[25] Bharath Sudharsan, John G. Breslin, and Muhammad Intizar Ali. Mlmcu: A framework to train ml classifiers on mcu-based iot edge devices. IEEE Internet of Things Journal, pages 1-1, 2021.

[26] Francesco Alongi, Nicolò Ghielmetti, Danilo Pau, Federico Terraneo, and William Fornaciari. Tiny neural networks for environmental predictions: An integrated approach with miosix. In 2020 IEEE International Conference on Smart Computing (SMARTCOMP), pages 350-355, 2020.

[27] Reddy T Chandrasekhara, Gadicherla Sirisha, and A Mallikarjuna Reddy. Smart healthcare analysis and therapy for voice disorder using cloud and edge computing. In 2018 4th International Conference on Applied and Theoretical Computing and Communication Technology (iCATccT), 2018.

[28] S. Y. Nikouei, C. Yu, S. Song, R. Xu, and T. R. Faughnan. Real-time human detection as an edge service enabled by a lightweight $\mathrm{cnn}$. IEEE, 2018.

[29] Wai-Chi Fang, Kai-Yen Wang, Nicolas Fahier, Yun-Lung Ho, and Yu-De Huang. Development and validation of an eeg-based real-time emotion recognition system using edge ai computing platform with convolutional neural network system-on-chip design. IEEE Journal on Emerging and Selected Topics in Circuits and Systems, 9(4):645-657, 2019.

[30] D. Gari, Clifford, Chengyu, Liu, Benjamin, Moody, H. Li-Wei, Lehman, Ikaro, and Silva. Af classification from a short single lead ecg recording: the physionet/computing in cardiology challenge 2017. 2017.

[31] Feifei Liu, Chengyu Liu, Lina Zhao, Xiangyu Zhang, and Eddie Ng Yin Kwee. An open access database for evaluating the algorithms of electrocardiogram rhythm and morphology abnormality detection. Journal of Medical Imaging and Health Informatics, 8(7):1368-1373, 2018.

[32] J. Pan and W. J. Tompkins. A real-time qrs detection algorithm. BME32(3):230-236, 1985

[33] P. V. Gent, H. Farah, N. V. Nes, and B. V. Arem. Heart rate analysis for human factors: Development and validation of an open source toolkit for noisy naturalistic heart rate data. In HUMANIST 2018 conference, 2018. 
[34] A. J Camm, M Malik, J. T Bigger, G Breithardt, S Cerutti, R. J Cohen, P Coumel, E. L Fallen, H. L Kennedy, and R. E Kleiger. Heart rate variability. standards of measurement, physiological interpretation, and clinical use. task force of the european society of cardiology and the north american society of pacing and electrophysiology. Circulation, 93(5):1043-65, 1996.

[35] I. Cygankiewicz and W. Zareba. Heart rate variability. Handb Clin Neurol, 117(4):379-393, 2013.

[36] A. Blum. Neural networks in C++:an object-oriented framework for building connectionist systems. Neural networks in C++:an objectoriented framework for building connectionist systems, 1992.

[37] A. Cutkosky and H. Mehta. Momentum improves normalized sgd. 2020.

[38] Ptd Boer, D. P. Kroese, S. Mannor, and R. Y. Rubinstein. A tutorial on the cross-entropy method. Annals of Operations Research, 134(1):1967, 2005.

[39] C. Ma, S. Wei, T. Chen, J Zhong, and C. Liu. Integration of results from convolutional neural network in a support vector machine for the detection of atrial fibrillation. IEEE Transactions on Instrumentation and Measurement. 